\title{
Dans le palais des glaces de la littérature romande, textes réunis par Vittorio Frigerio et Corine Renevey
}

Luigi Luison

\section{(2) OpenEdition}

Journals

\section{Edizione digitale}

URL: https://journals.openedition.org/studifrancesi/41470

DOI: $10.4000 /$ studifrancesi. 41470

ISSN: 2421-5856

\section{Editore}

Rosenberg \& Sellier

\section{Edizione cartacea}

Data di pubblicazione: 1 juillet 2004

Paginazione: 246

ISSN: 0039-2944

\section{Notizia bibliografica digitale}

Luigi Luison, «Dans le palais des glaces de la littérature romande, textes réunis par Vittorio Frigerio et Corine Renevey», Studi Francesi [Online], 142 (XLVIII | I) | 2004, online dal 30 novembre 2015, consultato il 09 septembre 2021. URL: http://journals.openedition.org/studifrancesi/41470; DOI: https://doi.org/10.4000/studifrancesi.41470

Questo documento è stato generato automaticamente il 9 septembre 2021.

\section{cc) (†) $\odot$}

Studi Francesi è distribuita con Licenza Creative Commons Attribuzione - Non commerciale - Non opere derivate 4.0 Internazionale. 


\title{
Dans le palais des glaces de la littérature romande, textes réunis par Vittorio Frigerio et Corine Renevey
}

\author{
Luigi Luison
}

\section{NOTIZIA}

AA. VV., Dans le palais des glaces de la littérature romande, textes réunis par Vittorio FRIGERIO et Corine RENEVEY, Amsterdam, éditions Rodopi, 2002, pp. 168.

1 I testi riuniti in questo volume sono il frutto delle attività svolte al Centre de documentation et de recherches sur les littératures romandes dell'Università di Toronto tra il 1995 ed il 1999. Lo scopo di questi studi, diretti da Vittorio Frigerio e Corine RENEVEY, è volta all'approfondimento dei rapporti culturali tra il Canadà e la Svizzera.

Gli scritti di questa raccolta, che provengono sia da autori romandi che da critici nordamericani interessati ai vari aspetti della letteratura di produzione romanda, convergono in un contesto unitario che rende possibile la convivenza di individualità diverse in una comune consapevolezza culturale.

Il primo ad affrontare la tematica proposta da questo volume è Jérôme Meizoz (Une langue perdue), il cui lavoro riguarda l'importanza della lingua e dei dialetti regionali collocandoli in un contesto prevalentemente emotivo. Strettamente tecnico, invece, è il saggio di Monique LAEDERACH (Traductions du pays, traductions de France: quelles qualités) che, presentando un panorama sulla traduzione letteraria elvetica, evidenzia la necessità di intensificare la produzione culturale della Svizzera romanda attraverso gli scambi culturali. Sulla produzione di Jacques CHESSEX si sofferma David J. Bond (Jacques Chessex: la Nature vue par un calviniste) che ne analizza la scrittura, caratterizzata da un conflitto tra Natura, calvinismo e principio di libertà. Un altro aspetto della letteratura romanda è rappresentato dagli scrittori figli di immigrati, e in questo contesto si inserisce il lavoro di Habiba SEBKHI (L'après-origines ou l'identité rhizomatique) che afferma 
la necessità di una «riconciliazione tra mondi e culture diversi tra loro. Il testo di Claire-Lise TONDEUR (Marginalité artistique, malaise identitaire et l'écriture romande) si concentra, invece, sulla crisi d'identità degli scrittori romandi e propone un riavvicinamento tra la Svizzera e i suoi autori. Questo argomento introduce i testi di Claude DARBELLAY (Blanche-neige et les cinq nains e Écrire en Suisse: le cinquième point cardinal) che si focalizzano sui rapporti tra letteratura e società, in un ambito che vede l'utilità sociale dello scrittore. Hélène BEZENçoN (Le jeu d'écrire, e La parole ou la bourse) evidenzia il rapporto fra leggenda e scrittura; secondo l'A. il compito della scrittura è quello di sottrarre la leggenda alla sua dimensione di forza irrazionale e «naturale». Jean ROMAN (C'est parce que je suis d'ici que je suis d'ailleurs) parla di radici e di conoscenza delle proprie origini; secondo l'A. questo aspetto costituisce il mezzo che permette all'individuo di aprirsi verso gli altri «sans nous perdre dans le néant de l'indéfini». Infine, Michel GoELDLIN (Pour tutoyer les anges), utilizzando uno stile autobiografico, porta l'attenzione del lettore sul fascino del viaggio, le cui caratteristiche rendono possibile un tipo di scrittura che va al di là della specificità dell'individuo. Il presente volume si chiude con una ricca bibliografia, a cura di Corine RENEVEY, sulla letteratura romanda. 